\title{
UNIQUENESS IN THE CAUCHY PROBLEM FOR PARABOLIC EQUATIONS ${ }^{1}$
}

\author{
BY
}

\author{
ROGER M. HAYNE
}

\begin{abstract}
In a classical paper S. Täcklind (Nova Acta Soc. Sci. Upsaliensis (4) 10 (1936), 1-57) closed the uniqueness question for the Cauchy problem for the heat equation with a general growth hypothesis which was both necessary and sufficient. Täcklind's proof of the sufficiency involved an ingenious bootstrapping comparison technique employing the maximum principle and a comparison function constructed from the Green's function for a half cylinder. G. N. Zolotarev (Izv. Vysš. Učebn. Zaved. Matematika 2 (1958), 118-135) has extended this result using essentially the same technique to show that Täcklind's uniqueness condition remains sufficient for a general second order parabolic equation provided the coefficients are regular enough to permit the existence and estimation of a Green's function. We have now shown, using a new approach which replaces the construction based upon a Green's function by an appropriate comparison solution of the maximizing equation (C. Pucci, Ann. Mat. Pura Appl. 72 (1966), 141-170), that Täcklind's condition is sufficient without any regularity conditions on the coefficients.
\end{abstract}

I. Introduction. In this paper we are concerned with the question of uniqueness of solutions of the initial value problem

$$
\begin{cases}L u-\partial u / \partial t=f & \text { in } H=\mathbf{R}^{n} \times(0, T), \\ u(x, 0)=\varphi(x) & \text { for } x \in \mathbf{R}^{n},\end{cases}
$$

where $0<T \leqslant \infty$ and $L$ is a second order elliptic operator of the form

$$
L=\sum_{i j=1}^{n} a_{i j}(x, t) \frac{\partial^{2}}{\partial x_{i} \partial x_{j}}+\sum_{i=1}^{n} b_{i}(x, t) \frac{\partial}{\partial x_{i}}+c(x, t)
$$

with variable coefficients $a_{i j}, b_{i}$ and $c$ defined in $H$. It suffices, by linearity, to consider only the homogeneous case $f \equiv \varphi \equiv 0$. It is well known in fact ([5], [6], [7], [9]) that without additional restrictions on the solution function $u$

Received by the editors December 2, 1975 and, in revised form, April 15, 1977.

AMS (MOS) subject classifications (1970). Primary 35K15; Secondary 35B30, 35R05.

Key words and phrases. Cauchy problem, uniqueness, extremal operators, second order parabolic equations, partial differential equations, maximum principle, a priori estimate, Bessel functions.

'This work represents a portion of the author's Doctoral Dissertation presented in August, 1975, at the University of California at Riverside. 
uniqueness will fail, but that in certain cases it can be restored by imposing a growth condition as $|x|^{2}=\sum_{i=1}^{n} x_{i}^{2} \rightarrow \infty$.

For the simplest case, that of the heat equation

$$
\sum_{i=1}^{n} \frac{\partial^{2} u}{\partial x_{i}^{2}}=\frac{\partial u}{\partial t}
$$

Tychonoff [7] has shown that uniqueness holds within the class of solutions such that

$$
|u(x, t)| \leqslant M \exp \left(\mu|x|^{2}\right) \quad \text { for }(x, t) \in H
$$

where $M, \mu \geqslant 0$ are constant. Holmgren [2] has relaxed this condition to

$$
|u(x, t)| \leqslant M \exp \left(\mu|x|^{2} \log |x|\right) .
$$

On the other hand, given $\varepsilon>0$, Tychonoff has constructed a nontrivial solution of the homogeneous problem such that

$$
|u(x, t)| \leqslant M \exp \left(\mu|x|^{2+\varepsilon}\right) \text { for }(x, t) \in H .
$$

Finally Täcklind [6] completely closed this question for the heat equation by showing that in the class of solutions $u$ such that for some constant $\mu>0$,

$$
|u(x, t)| \leqslant \exp (\mu|x| p(|x|)) \quad \text { for }(x, t) \in H,|x|>1
$$

where $p(r)$ is some given positive continuous function, uniqueness holds if and only if

$$
\int^{\infty} \frac{d r}{\bar{p}(r)}=+\infty \quad\left(\bar{p}(r)=\inf _{r<s} p(s)\right)
$$

To establish the sufficiency of (1.5) Täcklind used the Green's function for the cylinder $(|x|<l, 0<t<T)$ to obtain an estimate for any solution there in terms of its maximum value on the bottom $(|x| \leqslant l, t=0)$, its maximum on the sides $(x=l, 0<t \leqslant T)$, and an exponential factor. With this crucial estimate, (1.5), and an ingenious boot strapping argument he has shown that a solution of the homogeneous problem which satisfies (1.4) must be arbitrarily small. Necessity is demonstrated by both Täcklind and Tychonoff with the explicit construction of a nontrivial solution of the homogeneous problem using the theory of quasianalytic functions (see [1]).

Generalizing in another direction Krzyański [3] showed that the Tychonoff condition (1.3) also is sufficient to guarantee uniqueness for operators $L$ of the from (1.2) provided the coefficients are bounded and continuous. Using techniques similar to those of Täcklind, Zolotarev [9] improved Krzyański's results by showing that (1.5) is a sufficient condition to give uniqueness of solutions of (1.1) in the class (1.4) if the coefficients are sufficiently regular (for example $C^{2+\alpha}$ and bounded). These regularity conditions are essential to 
assure the existence of a Green's function from which Zolotarev, like Täcklind, derived the crucial estimates. In the case that the coefficients of $L$ are constant, Zolotarev constructed a counterexample, like that of Täcklind, to show that (1.5) is necessary. It should be noted that Zolotarev actually worked with systems of equations of which our problem is a special case.

Our main purpose in this paper is to establish, with conditions only on the magnitudes of the coefficients, the following:

THEOREM. Solutions of (1.1) in the class (1.4) with the function $r p(r)$ nondecreasing are unique provided the Täcklind condition (1.5) holds.

A precise statement, in more general form, is given in §II, in an extended maximum principle (Theorem III) and its corollaries. We stress here that all conditions on the regularity of the coefficients have been removed, which is of some significance for nonlinear problems. Our proof includes the use of a modified version of Täcklind's bootstrapping argument. Our principal innovation, however, is the use of the recent theory of elliptic extremal operators (see [4]) to obtain a comparison function independent of coefficient regularity which is then used to derive the crucial estimates. This is in contrast to Täcklind and Zolotarev who depend upon a Green's function for this purpose, thus restricting themselves to regular coefficients.

We define our notation and basic hypothesis, state the principal results and prove all but the most difficult (Theorems I and II) in §II. In §III we introduce extremal operators and outline their application to our problem. We then construct our comparison function and establish some of its properties (Theorem III). The proof of our modification of Täcklind's basic comparison theorem (Theorem I) is given in §IV.

I would like to take this opportunity to express my gratitude to my advisor, Professor J. K. Oddson, whose help and guidance was invaluable in this work.

II. Notation and basic results. We shall denote by $x=\left(x_{1}, \ldots, x_{n}\right)$ a point in $\mathbf{R}^{n}, n \geqslant 1$, by $|x|$ the usual Euclidean norm

$$
|x|=\left(\sum_{i=1}^{n} x_{i}^{2}\right)^{i / 2},
$$

and by $\Omega$ a fixed open subset of $\mathbf{R}^{n} \times(0, \infty)$. In $\Omega$ we shall consider second order parabolic partial differential operators of the form

$$
L=\sum_{i j=1}^{n} a_{i j}(x, t) \frac{\partial^{2}}{\partial x_{i} \partial x_{j}}+\sum_{i=1}^{n} b_{i}(x, t) \frac{\partial}{\partial x_{i}}+c(x, t) \cdot-d(x, t) \frac{\partial}{\partial t}
$$

with coefficients $a_{i j}, b_{i}, c$ and $d$ which are defined and satisfy the following conditions for all $(x, t) \in \Omega$ : 
(i) $\sum_{i j=1}^{n} a_{i j}(x, t) \xi_{i} \xi_{j}>\alpha|\xi|^{2}$ for all $\xi \in \mathbf{R}^{n}$,

(ii) $\sum_{i=1}^{n} a_{i i}(x, t) \equiv 1$,

(iii) $b_{i}$ are bounded and $\left(\sum_{i=1}^{n} b_{i}^{2}\right)^{1 / 2} \leqslant \beta /|x|$,

(iv) $d(x, t) \geqslant k>0$,

(v) $c(x, t) \leqslant c_{0}$.

Here $\alpha, \beta, k$ and $c_{0}$ are some fixed constants satisfying $\alpha, \beta \geqslant 0$ and $k>0$. In this and other sections we will need the additional constants $h=1-(n-$ 1) $\alpha$ and $\nu=\frac{1}{2}\left(1-h^{-1}(1+\beta-h)\right)$.

REMARK 1. The conditions (i)-(v) are solely on the magnitudes of the coefficients of (2.1). There are no regularity assumptions. More generally, in many of our results we could, in fact, permit the operator $L$ to be quasilinear with coefficients which also involve the solution function $u$ and its derivatives provided that these coefficients, as functions of $x$ and $t$ after insertion of the particular function $u$, satisfy (i)-(v).

REMARK 2. Condition (ii) will be a convenient normalization. It can be achieved for any uniformly parabolic operator of the form

$$
\begin{aligned}
L= & \sum_{i j=1}^{n} A_{i j}(x, t) \frac{\partial^{2}}{\partial x_{i} \partial x_{j}} \\
& +\sum_{i=1}^{n} B_{i}(x, t) \frac{\partial}{\partial x_{i}}+C(x, t) \cdot-\frac{\partial}{\partial t}
\end{aligned}
$$

with bounded coefficients by dividing by the positive function $\sum_{i=1}^{n} A_{i i}$. Note that (i) and (ii) imply that $0 \leqslant \alpha \leqslant 1 / n, 1 / n \leqslant h \leqslant 1$ and $\nu \leqslant 1 / 2$.

REMARK 3. It is well known that operators of the form (2.1) enjoy the weak maximum principle, i.e. any sufficiently regular solution of $L u \geqslant 0$ in a bounded domain must attain its maximum on the "parabolic boundary" of that domain. More precisely, if $\bar{n}(x, t)=\left(n_{x}(x, t), n_{t}(x, t)\right)$ denotes the unit outer normal for $(x, t) \in \partial \Omega$ when it exists and if

$$
E=\{(x, t) \in \partial \Omega: \bar{n}(x, t) \text { exists and } \bar{n}(x, t)=(0,1)\},
$$

then the parabolic boundary of $\Omega$ is given by

$$
\partial_{p} \Omega=\overline{(\partial \Omega) \backslash E} .
$$

The statements and proofs of our results will require the definition of several sets.

Definition 2.1. For $l>1$ and $t_{0} \geqslant 0$ we define:

$$
\begin{aligned}
D\left(l, t_{0}\right)=\left\{(x, t) \in \mathbf{R}^{n} \times(0, \infty):|x|<\right. & l, t_{0}<t<t_{0} \\
& \left.+\min \left(\frac{k\left(l^{2}-1\right)}{8 h(2-\nu)}, \frac{k(l-|x|)^{2}}{8 h(2-\nu)}\right)\right\},
\end{aligned}
$$




$$
\begin{aligned}
& P\left(l, t_{0}\right)=\left\{(x, t) \in \mathbf{R}^{n} \times(0, \infty): 1 \leqslant|x|<l, t=t_{0}+\frac{k(l-|x|)^{2}}{8 h(2-\nu)}\right\}, \\
& \Omega\left(l, t_{0}\right)=\Omega \cap D\left(l, t_{0}\right), \\
& \Gamma\left(l, t_{0}\right)=\left\{\left(x, t_{0}\right) \in \partial \Omega\left(l, t_{0}\right):|x| \leqslant l\right\}, \\
& S\left(l, t_{0}\right)=\left(\overline{\Omega \cap P\left(l, t_{0}\right)}\right) \backslash\left(\Gamma\left(l, t_{0}\right) \cup \partial_{p} \Omega\right), \\
& \delta\left(l, t_{0}\right)=\left(\partial_{p} \Omega\right) \cap\left[\overline{D\left(l, t_{0}\right)} \backslash\left(\Gamma\left(l, t_{0}\right) \cup S\left(l, t_{0}\right)\right)\right] .
\end{aligned}
$$

REMARK 1. The domain $D\left(l, t_{0}\right)$ takes the place of the cylinder $Q\left(l, t_{0}\right)=$ $\left\{(x, t):|x|\langle l, t\rangle t_{0}\right\}$ in which Täcklind and Zolotarev use the Green's function. The unusual shape of $D\left(l, t_{0}\right)$ is due to the character of the comparison function which we construct (see §III).

REMARK 2. Let us note that $\partial_{p} \Omega\left(l, t_{0}\right)=S\left(l, t_{0}\right) \cup \Gamma\left(l, t_{0}\right) \cup \delta\left(l, t_{0}\right)$. In the particular case $\Omega \equiv H \equiv \mathbf{R}^{n} \times(0, T)$ and $0 \leqslant t_{0}<T \leqslant \infty$ then $\Omega\left(l, t_{0}\right)$ and $S\left(l, t_{0}\right)$ are the portions of $D\left(l, t_{0}\right)$ and $P\left(l, t_{0}\right)$, respectively, with $t<T$, $\delta\left(l, t_{0}\right)=\varnothing$ and $\Gamma\left(l, t_{0}\right)$ is the bottom of $D\left(l, t_{0}\right)$.

Our proofs will use Täcklind's technique with modifications to handle the more complicated sets with which we are working. One particular type of estimate appears repeatedly in a bootstrapping argument which eventually leads to the conclusion that the solution function is arbitrarily small. We extract this critical property in the form:

Definition 2.2 (Property (T)). We say that a real valued function $u$ has property $(\mathrm{T})$ in a set $D$ if for each $\Omega\left(l, t_{0}\right)$ contained in $D$ the two conditions

$$
\begin{gathered}
\limsup _{\substack{(x, t) \rightarrow \Gamma\left(l, t_{0}\right) \\
(x, t) \in \Omega\left(l, t_{0}\right)}} u(x, t) \leqslant K_{1}, \\
\limsup _{\substack{(x, t) \rightarrow S\left(l, t_{0}\right) \\
(x, t) \in \Omega\left(l, t_{0}\right)}} u(x, t) \leqslant K_{1}+K_{2} \quad\left(K_{1}, K_{2} \geqslant 0\right)
\end{gathered}
$$

imply that

$$
u(x, t) \leqslant K_{1}+C K_{2} \exp \left(-\frac{k(l-|x|)^{2}}{8 h\left(t-t_{0}\right)}\right)
$$

for all $(x, t) \in \Omega\left(l, t_{0}\right)$, where $C$ is some constant independent of the choice of $l$ or $t_{0}$.

We are now ready to state the main results of this paper:

TheOREM I (COMPARISON THEOREM). Suppose $p(r)$ is a positive, continuous, real valued function defined for $r>1$ with $r p(r)$ nondecreasing such that

$$
\int^{\infty} \frac{d r}{p(r)}=+\infty
$$


Let $T, \varepsilon>0, M, \mu \geqslant 0$ and $l>1$ be given. Then there is a real number $l^{*}>l$ such that the conditions

(1) $u$ has property $(\mathrm{T})$ in $\Omega\left(l^{*}, 0\right)$,

(2) $\lim \sup _{(x, t) \rightarrow \Gamma\left(l^{*}, 0\right) \cup \delta\left(l^{*}, 0\right) ;(x, t) \in \Omega\left(l^{*}, 0\right)} u(x, t) \leqslant 0$,

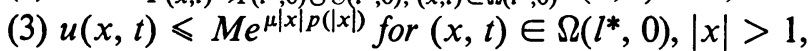
imply that $u(x, t)<\varepsilon$ for all $(x, t) \in \Omega\left(l^{*}, 0\right)$ with $|x|<l$ and $t<T$.

We shall prove this theorem in §IV. There we will also see that under our assumption that $r p(r)$ is nondecreasing the Täcklind condition (1.5) and our seemingly stronger condition $\int^{\infty} p^{-1}(r) d r=+\infty$ are actually equivalent. Since $\varepsilon$ can be arbitrarily small, Theorem I shows the question of uniqueness in the initial value problem (1.1) can be reduced to proving that solutions satisfy property $(\mathrm{T})$ in the sets $\Omega\left(l^{*}, 0\right)$. Täcklind and Zolotarev used a Green's function in the simpler domains $Q\left(l, t_{0}\right)$ for this purpose whereas we accomplish it with the aid of a comparison function having the following properties in $D\left(l, t_{0}\right)$ :

THEOREM II. Let $L$ be an operator (2.1) satisfying (i)-(v) with $c_{0} \leqslant 0$. For any $l>1$ and $t_{0} \geqslant 0$, there exists a nonnegative function $v$ such that

(II-1) $v$ is twice continuously differentiable with respect to the $x$ variables and once with respect to $t$ in $D\left(l, t_{0}\right)$ and $v$ is continuous in $\overline{D\left(l, t_{0}\right)} \backslash\left\{\left(x, t_{0}\right)\right.$ : $|x|=l\}$,

(II-2) $L v \leqslant 0$ in $D\left(l, t_{0}\right)$

(II-3) $v\left(x, t_{0}\right)=0$ for $|x|<l$,

(II-4) $v(x, t) \geqslant B>0$ on the parabolic sides $P\left(l, t_{0}\right)$,

(II-5) $v(x, t) \leqslant A \exp \left(-k(l-|x|)^{2} / 8 h\left(t-t_{0}\right)\right)$ in $D\left(l, t_{0}\right)$.

Here $A$ and $B$ are positive constants depending only upon the operator $L$ (in fact only on the constants $\alpha, \beta$ and $n$ ).

The proof of this theorem will also be deferred, to be given in §III in a series of lemmas.

With the aid of these fundamental theorems (I and II) we can now establish our main results. Applying first Theorem II we have

LEMMA 2.1. Let $L$ be an operator (2.1) satisfying (i)-(v) with $c_{0} \leqslant 0$. Suppose $u(x, t)$ is a function, differentiable with respect to $t$ and twice differentiable with respect to $x$ in $\Omega$, such that

$$
L u \geqslant 0 \quad \text { in } \Omega, \quad \limsup _{\substack{(x, t) \rightarrow \partial_{\rho} \Omega \\(x, t) \in \Omega}} u(x, t) \leqslant 0 .
$$

Then $u$ has property $(\mathrm{T})$ in $\Omega\left(l^{*}, 0\right)$ for any $l^{*}>1$.

Proof. Let $l>1$ and $t_{0} \geqslant 0$ be such that $\Omega\left(l, t_{0}\right) \subseteq \Omega\left(l^{*}, 0\right)$. Suppose that $K_{1}, K_{2} \geqslant 0$ are constants such that 


$$
\begin{aligned}
& \limsup _{\substack{(x, t) \rightarrow \Gamma\left(l, t_{0}\right) \\
(x, t) \in \Omega\left(l, t_{0}\right)}} u(x, t) \leqslant K_{1} \quad \text { and } \\
& \quad \lim _{\substack{(x, t) \\
(x, t) \rightarrow S\left(l, t_{0}\right) \\
(x, t) \in \Omega\left(l, t_{0}\right)}} u(x, t) \leqslant K_{1}+K_{2}
\end{aligned}
$$

and consider the function

$$
\omega(x, t)=K_{1}+K_{2} v(x, t) / B-u(x, t)
$$

in $\Omega\left(l, t_{0}\right)$, where $v$ is the comparison function and $B$ the constant given in Theorem II. We note that

$$
L \omega=c K_{1}+K_{2} L v / B-L u \leqslant c_{0} K_{1} \leqslant 0 \text { in } \Omega\left(l, t_{0}\right) .
$$

Moreover we have

$$
\begin{aligned}
& \liminf _{\substack{x, t) \rightarrow \Gamma\left(l, t_{0}\right) \\
(x, t) \in \Omega\left(l, t_{0}\right)}} \omega(x, t) \geqslant K_{1}-\lim _{\substack{(x, t) \rightarrow \Gamma\left(l, t_{0}\right) \\
(x, t) \in \Omega\left(l, t_{0}\right)}} u(x, t) \geqslant 0, \\
& \liminf _{\substack{(x, t) \rightarrow \delta\left(l, t_{0}\right) \\
(x, t) \in \Omega\left(l, t_{0}\right)}} \omega(x, t) \geqslant-\lim _{\substack{(x, t) \rightarrow \delta\left(l, t_{0}\right) \\
(x, t) \in \Omega\left(l, t_{0}\right)}} u(x, t) \geqslant 0
\end{aligned}
$$

and

$$
\begin{aligned}
\liminf _{\substack{(x, t) \rightarrow S\left(l, t_{0}\right) \\
(x, t) \in \Omega\left(l, t_{0}\right)}} \omega(x, t) & \geqslant \lim _{\substack{(x, t) \rightarrow S\left(l, t_{0}\right) \\
(x, t) \in \Omega\left(l, t_{0}\right)}}\left(K_{1}+\frac{K_{2}}{B} v(x, t)\right)-\limsup _{\substack{(x, t) \rightarrow S\left(l, t_{0}\right) \\
(x, t) \in \Omega\left(l, t_{0}\right)}} u(x, t) \\
& \geqslant\left(K_{1}+K_{2}\right)-\left(K_{1}+K_{2}\right)=0 .
\end{aligned}
$$

From the weak maximum principle, since $\partial_{p} \Omega\left(l, t_{0}\right)=\Gamma\left(l, t_{0}\right) \cup S\left(l, t_{0}\right) \cup$ $\delta\left(l, t_{0}\right)$ we conclude that $\omega \geqslant 0$ in $\Omega\left(l, t_{0}\right)$. Using (II-5) we then obtain

$$
u(x, t) \leqslant K_{1}+C K_{2} \exp \left(-\frac{k(l-|x|)^{2}}{8 h\left(t-t_{0}\right)}\right) \text { in } \Omega\left(l, t_{0}\right)
$$

which establishes the result.

We now combine Theorem I and Lemma 2.1 to obtain our major result:

Theorem III (EXTENDEd MAXIMUM PRINCIPLE). Let $L$ be an operator (2.1) satisfying (i)-(v). Suppose $u(x, t)$ is a function, differentiable with respect to $t$ and twice differentiable with respect to $x$ in $\Omega$, such that

$$
\begin{gathered}
L u \geqslant 0 \quad \text { in } \Omega, \\
\underset{\substack{(x, t) \rightarrow \sup _{p} \Omega \\
(x, t) \in \Omega}}{\lim \sup }(x, t) \leqslant 0 .
\end{gathered}
$$

If, in addition, there is a positive, continuous function $p(r)$ defined for $r>1$ with $r p(r)$ nondecreasing such that

$$
u(x, t) \leqslant M \exp [\mu|x| p(|x|)] \text { for }(x, t) \in \Omega,|x|>1
$$


for some constants $M, \mu>0$ and

$$
\int^{\infty} \frac{d r}{p(r)}=+\infty
$$

then $u(x, t)<0$ in $\Omega$.

Proof. Suppose first that $c_{0}<0$. Fix $\left(x^{*}, t^{*}\right) \in \Omega$ and let $\varepsilon>0$ be given. Choose $l>\max \left(1,\left|x^{*}\right|\right), T>t^{*}$ and let $l^{*}$ be the constant given by Theorem I. Since, by Lemma $2.1, u$ has property $(T)$ in $\Omega\left(l^{*}, 0\right)$, we can apply Theorem I to obtain $u\left(x^{*}, t^{*}\right)<\varepsilon$. The conclusion follows since $\varepsilon>0$ and $\left(x^{*}, t^{*}\right) \in$ $\Omega$ were arbitrary.

If now $c_{0}>0$ we consider the function $\omega(x, t)=\exp \left[-c_{0} t / k\right] u(x, t)$, noting that $L^{*} \omega>0$ in $\Omega$, where

$$
L^{*}=L-\frac{c_{0}}{k} d=\sum_{i j=1}^{n} a_{i j} \frac{\partial^{2}}{\partial x_{i} \partial x_{j}}+\sum_{i=1}^{n} b_{i} \frac{\partial}{\partial x_{i}}+\left(c-\frac{c_{0}}{k} d\right) \cdot-d \frac{\partial}{\partial t}
$$

is an operator of the form (2.1) satisfying (i)-(v) with

$$
c-c_{0} d / k<c-c_{0}<0 \text {. }
$$

Clearly we have

$$
\lim _{\substack{(x, t) \rightarrow \partial_{p} \Omega \\(x, t) \in \Omega}} \omega(x, t)<0
$$

and, for $(x, t) \in \Omega,|x|>1$,

$$
\omega(x, t)<M \exp [\mu|x| p(|x|)] .
$$

We may now conclude, using the first part of the proof, that $\omega$, and thus $u$, is nonpositive in $\Omega$.

REMARK. Replacing $u$ by $-u$ in Theorem III gives the usual companion result which we do not state.

As a useful application of Theorem III we have

COROLlaRY 1 (A PRIORI ESTIMATE). Let $L$ be an operator of the form (2.1) satisfying (i)-(v) with $c_{0}<0$. Suppose $u(x, t)$ is a function, differentiable with respect to $t$, twice differentiable with respect to $x$ in $\Omega$ and continuous in $\bar{\Omega}$, such that, for some constants $M, \mu<0$,

$$
|u(x, t)|<M \exp [\mu|x| p(|x|)] \text { for }(x, t) \in \Omega,|x|>1
$$

with $p$ as in Theorem III. If, for $0<T<\infty$, we set $\Omega_{T}=\{(x, t) \in \Omega$ : $t<T\}$, then we have the estimate

$$
|u(x, t)|<\sup _{\partial_{p} \Omega_{T}}|u(x, t)|+\frac{t}{k} \sup _{\Omega_{T}}|L u(x, t)| \quad \text { for all }(x, t) \in \Omega_{T} .
$$

Proof. For ease of notation, set 


$$
A=\sup _{\partial_{p} \Omega_{T}}|u(x, t)| \text { and } B=\sup _{\Omega_{T}}|L u(x, t)| .
$$

If either $A$ or $B$ is infinite there is nothing to prove; otherwise, consider the functions

$$
\omega=A+t B / k \pm u \text { in } \Omega_{T} .
$$

We have

$$
\begin{gathered}
L \omega=c A-d B / k \pm L u \leqslant-B \pm L u \leqslant 0 \text { in } \Omega_{T}, \\
\omega=A+t B / k \pm u \geqslant A \pm u \geqslant 0 \text { on } \partial_{p} \Omega_{T}
\end{gathered}
$$

and finally, in $\Omega_{T}$,

$$
\begin{aligned}
\omega(x, t) & \geqslant A+t B / k-|u(x, t)| \geqslant-|u(x, t)| \\
& >-M \exp [\mu(x) p(|x|)] .
\end{aligned}
$$

Using the remark after Theorem III we may conclude that $\omega \geqslant 0$ in $\Omega_{T}$ which completes the proof.

REMARK. If $c_{0}>0$ we may obtain the corresponding estimate

$$
|u(x, t)| \leqslant \exp \left[\frac{c_{0}}{k} t\right]\left(\sup _{\partial_{\rho} \Omega_{T}}|u(x, t)|+\frac{t}{k} \sup _{\Omega_{T}}|L u(x, t)|\right) \quad \text { in } \Omega_{T}
$$

by considering the function $\omega=\exp \left[-c_{0} t / k\right] u$ as in Theorem III.

Uniqueness and continuous dependence on the data now follow easily.

COROLlaRY 2. Let $L$ be an operator (2.1) satisfying (i)-(v). Suppose $f$ and $\varphi$ are functions defined on $\Omega$ and $\partial_{p} \Omega$ respectively. In the class of functions $u$, differentiable with respect to $t$, twice differentiable with respect to $x$ in $\Omega$ and continuous in $\Omega \cup \partial_{p} \Omega$ such that $|u(x, t)| \leqslant M[\exp \mu|x| p(|x|)]$ for some constants $M, \mu>0$ and all $(x, t) \in \Omega,|x|>1$, with $p$ as in Theorem III, solutions of the problem

$$
\begin{aligned}
L u & =f \quad \text { in } \Omega, \\
u & =\varphi \quad \text { on } \partial_{p} \Omega,
\end{aligned}
$$

when they exist, are unique and depend continuously on the data, $f$ and $\varphi$, in the supremum norm in $\Omega_{T}$ for any $0<T<\infty$.

REMARK. As a special case this establishes the result mentioned in the Introduction for the slab $H=\mathbf{R}^{n} \times(0, T)$.

It should be noted, in conclusion, that Theorem III and Corollary 1 also apply in the case when $L$ is quasilinear in the sense that was mentioned earlier in this section. On the other hand, Corollary 2 does not apply without additional assumptions on the operator $L$, since the proof makes explicit use of linearity. For the homogeneous problem $f \equiv \varphi \equiv 0$, however, uniqueness still follows directly from Corollary 1. 
III. The comparison function and proof of Theorem II. In this section we construct our comparison function and verify its properties as given in Theorem II. For a fixed $t_{0} \geqslant 0$ and $l>1$ the function we wish to consider for this purpose is given in the cylinder $Q\left(l, t_{0}\right)=\left\{(x, t) \in \mathbf{R}^{n} \times(0, \infty):|x|<l\right.$, $t>t_{0}$ \} by the formula

$$
\begin{aligned}
v(x, t)= & \sqrt{k / 2 h} l^{1-\nu}|x|^{\nu} \\
& \times \int_{0}^{t-t_{0}} \frac{\exp \left(-k\left(l^{2}+|x|^{2}\right) / 4 h \tau\right)}{\tau \sqrt{t-t_{0}-\tau}} I_{-\nu}\left(\frac{k l|x|}{2 h \tau}\right) d \tau
\end{aligned}
$$

with $v\left(x, t_{0}\right)=0$ for $|x|<l$. Here the constants $k, h$ and $\nu$ are those defined in $\S$ II and $I_{-\nu}$ denotes the modified Bessel function of the first kind of order $-\nu$.

Before establishing the necessary properties of (3.1) we would like to give some motivation for its choice. Täcklind and Zolotarev, making use of the Green's function for their regular operator $L$, obtained their comparison function $v$ as a solution of $L v=0$ in $Q\left(l, t_{0}\right)$ satisfying the boundary conditions $v\left(x, t_{0}\right)=0$ for $|x|<l$ and $v(x, t) \geqslant 1$ for $|x|=l, t>t_{0}$. This leads us to seek our comparison function with the same boundary conditions but satisfying, instead, $L v \leqslant 0$ in $Q\left(l, t_{0}\right)$ since existence of solutions of $L v=0$ is unknown in general for the operators $L(2.1)$ which we consider. The theory of extremal operators (see [4]), which we will outline here, plays a major role in the construction of (3.1) and in the proof of Theorem II.

Let us denote by $\mathscr{L}_{\alpha}$ the class of operators $L^{*}$ of the form

$$
L^{*}=\sum_{i j=1}^{n} a_{i j}(x, t) \frac{\partial^{2}}{\partial x_{i} \partial x_{j}}
$$

with coefficients $a_{i j}$ defined in $\Omega$ satisfying there the properties (i) and (ii) of §II. It is easy to see that the class $\mathcal{L}_{1 / n}$ contains only the operator $n^{-1} \sum_{i=1}^{n}\left(\partial^{2} / \partial x_{i}^{2}\right)$, that $\alpha^{\prime} \leqslant \alpha$ implies $\varrho_{\alpha} \subseteq \varrho_{\alpha^{\prime}}$, and that $\varrho_{0}$ contains all operators that are elliptic or parabolic in $\Omega$ with respect to the $x$ variables. Pucci [4] has defined the maximizing operator $M_{\alpha}$ for the class $\mathcal{L}_{\alpha}$ by

$$
M_{\alpha}[u](x, t)=\sup _{L^{*} \in \mathfrak{L}_{\alpha}} L^{*} u(x, t)
$$

for any function $u$ defined in $\Omega$ which is twice differentiable with respect to the $x$-variables, and has obtained the representation

$$
M_{\alpha}[u]=\alpha \sum_{i=1}^{n} \frac{\partial^{2}}{\partial x_{i}^{2}}+(1-n \alpha) C_{n}[u]
$$

where $C_{n}[u]$ denotes the largest eigenvalue of the $n \times n$ Hessian matrix $\left(\partial^{2} u / \partial x_{i} \partial x_{j}\right)$. In the special case that $u$, as a function of $x$, depends on $r=|x|$ alone $M_{\alpha}[u]$ takes the simpler form 


$$
M_{\alpha}[u]=h_{1} u_{r r}+\left(1-h_{1}\right) u_{r} / r
$$

where

$$
h_{1}= \begin{cases}\alpha & \text { if } u_{r r}-u_{r} / r<0, \\ 1-(n-1) \alpha & \text { if } u_{r r}-u_{r} / r \geqslant 0 .\end{cases}
$$

In this section we use the maximizing operator $M_{\alpha}$ to give a systematic approach to the construction of (3.1). In fact, if the operator $L$ is of the form (2.1) satisfying (i) $-(v)$ with $c_{0} \leqslant 0$ we see that for any sufficiently smooth $v$, with $v_{t} \geqslant 0$,

$$
\begin{aligned}
L v & \leqslant M_{\alpha}[v]+\left|\sum_{i=1}^{n} b_{i} v_{x_{i}}\right|+c v-k\left|v_{t}\right| \\
& \leqslant M_{\alpha}[v]+\left(\sum_{i=1}^{n} b_{i}^{2}\right)^{1 / 2}|\operatorname{grad} v|+c v-k\left|v_{t}\right| \\
& \leqslant M_{\alpha}[v]+\beta|\operatorname{grad} v| /|x|+c v-k\left|v_{t}\right| .
\end{aligned}
$$

If, in particular, $v$ is a nonnegative function of $r=|x|$ and $t$ with $v_{r}, v_{t} \geqslant 0$ in $Q\left(l, t_{0}\right)$ then we have

$$
L v \leqslant h_{1} v_{r r}+\left(\beta+1-h_{1}\right) v_{r} / r-k v_{t}
$$

and the inequality $L v \leqslant 0$ will follow if $v$ can be chosen as a solution of the equation

$$
h_{1} v_{r r}+\left(\beta+1-h_{1}\right) v_{r} / r-k v_{t}=0 .
$$

The nonlinear dependence of $h_{1}$ on the derivatives of $v$ presents still another problem. If, however, $v_{r r}-v_{r} / r$ is of one sign, say nonnegative, in $Q\left(l, t_{0}\right)$ then $h_{1}=h=1-(n-1) \alpha$ is constant there. In summary the inequality $L v \leqslant 0$ will be satisfied for all our operators (2.1) by any sufficiently regular solution of the linear equation

$$
h v_{r r}+(\beta+1-h) v_{r} / r-k v_{t}=0
$$

having the properties that $v, v_{r}, v_{t}$ and $v_{r r}-v_{r} / r$ are all nonnegative. Our function (3.1) was obtained by Laplace transform techniques as a solution of this equation (3.4) under the conditions that $v\left(x, t_{0}\right)=0$ for $|x|<l$ and $\lim _{t \rightarrow t_{0}} v(x, t)=1$ for $|x|=l$.

We now turn to the justification of this procedure. In Lemmas 3.1 and 3.2 we show that the function $v$, given by (3.1), is well defined, nonnegative and is sufficiently regular in $Q\left(l, t_{0}\right)$. We then show, in Lemma 3.3 , that $v$ satisfies the linear equation (3.4). In order to justify the use of (3.4) instead of the nonlinear equation (3.3) we must verify that $v_{r r}-v_{r} / r \geqslant 0$. This inequality fails, in fact, near the sides of the cylinder $Q\left(l, t_{0}\right)$ but does hold (Lemma 3.4) in the subset $D\left(l, t_{0}\right)$ which was chosen for precisely this purpose. Finally the 
result (II-2), $L v \leqslant 0$ in $D\left(l, t_{0}\right)$, follows from Lemma $3.5\left(v_{t} \geqslant 0\right)$ and Lemma $3.6\left(v_{r} \geqslant 0\right)$.

As for the remainder of Theorem II, Lemma 3.2 contains (II-3), (II-4) is the result of Lemma 3.7 and property (II-5) is given by Lemma 3.8.

Before we state and prove these lemmas we shall need some properties of $I_{p}(r)$, the modified Bessel function of the first kind of order $p$ defined for the real variable $r>0$ and index $p \neq-1,-2,-3, \ldots$ by

$$
I_{p}(r)=\sum_{k=0}^{\infty} \frac{(r / 2)^{2 k+p}}{k ! \Gamma(k+p+1)},
$$

which can be found, for example, in $\$ \S 3.71$ and 7.23 of [8]. The asymptotic estimates

$$
\begin{aligned}
& I_{p}(r) \sim \frac{e^{r}}{\sqrt{2 \pi r}} \quad \text { as } r \rightarrow \infty, \text { and } \\
& I_{p}(r) \sim \frac{(r / 2)^{p}}{\Gamma(p+1)} \quad \text { as } r \rightarrow 0
\end{aligned}
$$

show that the function $r^{-p} e^{-r} I_{p}(r)(1+r)^{p+1 / 2}$ is a positive, uniformly bounded function of $r$ so that there are positive constants $\lambda_{p}$ and $\Lambda_{p}$, depending only upon $p$, such that

$$
\lambda_{p} \leqslant r^{-p} e^{-r} I_{p}(r)(1+r)^{p+1 / 2} \leqslant \Lambda_{p} \text { for } r \geqslant 0 .
$$

For $p>-\frac{1}{2}$ and $r>0$ we have the representation

$$
I_{p}(r)=\frac{(r / 2)^{p}}{\Gamma\left(p+\frac{1}{2}\right) \Gamma\left(\frac{1}{2}\right)} \int_{-1}^{1} e^{r \eta}\left(1-\eta^{2}\right)^{p-1 / 2} d \eta .
$$

Integration by parts in (3.7) yields

$$
I_{p+1}(r)=\frac{(r / 2)^{p}}{\Gamma\left(p+\frac{1}{2}\right) \Gamma\left(\frac{1}{2}\right)} \int_{-1}^{1} e^{r \eta} \eta\left(1-\eta^{2}\right)^{p-1 / 2} d \eta
$$

so that, for $p>-\frac{1}{2}$ and $r>0$, we have

$$
I_{p}(r)-I_{p+1}(r)=\frac{(r / 2)^{p}}{\Gamma\left(p+\frac{1}{2}\right) \Gamma\left(\frac{1}{2}\right)} \int_{-1}^{1} e^{r \eta}(1-\eta)\left(1-\eta^{2}\right)^{p-1 / 2} d \eta>0 .
$$

If $p=\frac{1}{2}$ then

$$
I_{p}(r)-I_{p+1}(r)=\sqrt{2 / \pi r}(\cosh r-\sinh r) \geqslant 0,
$$

and thus we obtain

$$
I_{p}(r)-I_{p+1}(r)>0 \text { for } r>0 \text { and } p \geqslant \frac{1}{2} .
$$

We will also make constant use of the following well-known relations 
between Bessel functions of different orders and their derivatives, valid for $r>0$ and $p \neq-1,-2, \ldots$ :

$$
\begin{aligned}
\frac{d}{d r}\left(r^{-p} I_{p}(c r)\right) & =c r^{-p} I_{p+1}(c r) \quad(c \text { constant }), \\
\frac{d}{d r}\left(r^{p} I_{p}(c r)\right) & =c r^{p} I_{p-1}(c r) \quad(c \text { constant }, p \neq 0), \\
\frac{d}{d r}\left(I_{p}(r)\right) & =I_{p+1}(r)+\frac{p}{r} I_{p}(r), \\
\frac{2 p}{r} I_{p}(r) & =I_{p-1}(r)-I_{p+1}(r) \quad(p \neq 0) .
\end{aligned}
$$

In the remainder of this section we will use the nonnegative constant $\gamma=h^{-1}(\beta+1-h)$ and the relations

$$
\nu=\frac{1}{2}(1-\gamma), \quad 1-\nu=\nu+\gamma=\frac{1}{2}(1+\gamma) .
$$

We note that, as a function of $t, v$ defined in (3.1) depends upon $t-t_{0}$ alone so that it suffices to consider only the case $t_{0}=0$. For convenience we set

$$
\begin{aligned}
g(r, t) & =t^{-1} \exp \left(-\frac{k\left(l^{2}+r^{2}\right)}{4 h t}\right) r^{\nu} I_{-\nu}\left(\frac{k l r}{2 h t}\right) \\
& =\left(\frac{k l}{2 h}\right)^{-\nu} t^{\nu-1} \exp \left(-\frac{k\left(l^{2}+r^{2}\right)}{4 h t}\right) \sum_{j=0}^{\infty} \frac{(k l r / 4 h t)^{2 j}}{j ! \Gamma(j+1-\nu)}
\end{aligned}
$$

for $r \geq 0$ and $t>0$ so that our comparison function (3.1) takes the form

$$
v(x, t)=\sqrt{\frac{k}{2 h}} l^{1-\nu} \int_{0}^{t} \frac{g(r, \tau)}{\sqrt{t-\tau}} d \tau, \quad r=|x| .
$$

LEMMA 3.1. The function $g(r, t)$ is analytic with respect to $r$ and $t$ and satisfies (3.4) for $r>0$ and $t>0$.

Proof. The regularity of $g$ is clear from its series representation in (3.14). In fact, $g$ is also an analytic function of $r^{2}$ for $t>0$ and hence $g_{r}(0, t)=0$ for $t>0$. It follows that $g_{r r}, g_{r} / r$ and $g_{t}$ are all continuous for $r \geqslant 0$ and $t>0$ so that it suffices to show $g$ satisfies (3.4) for $r, t>0$. In this case we can multiply (3.4) by $h^{-1} r^{\gamma}$ to obtain the equivalent equation

$$
\left(r^{\gamma} g_{r}\right)_{r}-k r^{\gamma} g_{t} / h=0 .
$$

A direct computation and (3.11) gives the formula 


$$
\begin{aligned}
g_{t}(r, t)= & r^{\nu} t^{-1} \exp \left(-\frac{k\left(l^{2}+r^{2}\right)}{4 h t}\right) \\
& \times\left\{\left(\frac{k\left(l^{2}+r^{2}\right)}{4 h t^{2}}-\frac{1}{t}\right) I_{-\nu}\left(\frac{k l r}{2 h t}\right)\right. \\
& \left.-\frac{k l r}{2 h t^{2}}\left[I_{1-\nu}\left(\frac{k l r}{2 h t}\right)-\frac{2 h t \nu}{k l r} I_{-\nu}\left(\frac{k l r}{2 h t}\right)\right]\right\}
\end{aligned}
$$

Combining terms and using (3.13) we obtain

$$
\begin{aligned}
g_{t}(r, t) & =r^{\nu} t^{-1} \exp \left(-\frac{k\left(l^{2}+r^{2}\right)}{4 h t}\right) \\
\times & \left\{\left(\frac{k\left(l^{2}+r^{2}\right)}{4 h t^{2}}-\frac{(1+\gamma)}{2 t}\right) I_{-\nu}\left(\frac{k l r}{2 h t}\right)-\frac{k l r}{2 h t^{2}} I_{1-\nu}\left(\frac{k l r}{2 h t}\right)\right\} .
\end{aligned}
$$

Another simple calculation gives

$$
\begin{aligned}
g_{r}(r, t)= & t^{-1} \exp \left(-\frac{k\left(l^{2}+r^{2}\right)}{4 h t}\right) \\
& \times\left\{\left[r^{\nu} I_{-\nu}\left(\frac{k l r}{2 h t}\right)\right]_{r}-\frac{k r^{\nu+1}}{2 h t} I_{-\nu}\left(\frac{k l r}{2 h t}\right)\right\},
\end{aligned}
$$

from which we obtain the representation

$$
\begin{aligned}
& {\left[r^{\gamma} g_{r}(r, t)\right]_{r}=} t^{-1} \exp \left(-\frac{k\left(l^{2}+r^{2}\right)}{4 h t}\right) \\
& \times\left\{\left(r^{\gamma}\left[r^{\nu} I_{-\nu}\left(\frac{k l r}{2 h t}\right)\right]_{r}\right)_{r}-\frac{k(\gamma+1)}{2 h t} r^{\nu+\gamma} I_{-\nu}\left(\frac{k l r}{2 h t}\right)\right. \\
& \quad-\frac{k r^{\gamma+1}}{2 h t}\left[r^{\nu} I_{-\nu}\left(\frac{k l r}{2 h t}\right)\right]_{r}-\frac{k r^{\gamma+1}}{2 h t}\left[r^{\nu} I_{-\nu}\left(\frac{k l r}{2 h t}\right)\right]_{r} \\
&\left.+\left(\frac{k r}{2 h t}\right)^{2} r^{\nu+\gamma} I_{-\nu}\left(\frac{k l r}{2 h t}\right)\right\} .
\end{aligned}
$$

We use (3.9) and then (3.10) with (3.13) to see that

$$
\begin{aligned}
\left(r^{\gamma}\left[r^{\nu} I_{-\nu}\left(\frac{k l r}{2 h t}\right)\right]_{r}\right)_{r} & =\frac{k l}{2 h t}\left[r^{\nu+\gamma} I_{1-\nu}\left(\frac{k l r}{2 h t}\right)\right]_{r} \\
& =\left(\frac{k l}{2 h t}\right)^{2} r^{\nu+\gamma} I_{-\nu}\left(\frac{k l r}{2 h t}\right),
\end{aligned}
$$


from which we obtain (3.15)

$$
\begin{aligned}
{\left[r^{\gamma} g_{r}(r, t)\right]_{r}=} & t^{-1} r^{\nu+\gamma} \exp \left(-\frac{k\left(l^{2}+r^{2}\right)}{4 h t}\right) \\
& \times\left\{\left[\frac{k^{2}\left(l^{2}+r^{2}\right)}{4 h^{2} t^{2}}-\frac{k(\gamma+1)}{2 h t}\right] I_{-\nu}\left(\frac{k l r}{2 h t}\right)\right. \\
= & \frac{k}{h} r^{\gamma} g_{t}(r, t) .
\end{aligned}
$$

Later we shall need the representation

$$
\begin{aligned}
g_{r r}(r, t)= & t^{-1} \exp \left(-\frac{k\left(l^{2}+r^{2}\right)}{4 h t}\right) \\
\times & \left\{\left[\left(\frac{k r}{2 h t}\right)^{2}-\frac{k}{2 h t}\right] r^{\nu} I_{-\nu}\left(\frac{k l r}{2 h t}\right)\right. \\
& +\left[\frac{k l}{2 h t}-2 l\left(\frac{k r}{2 h t}\right)^{2}\right] r^{\nu-1} I_{1-\nu}\left(\frac{k l r}{2 h t}\right) \\
& \left.+\left(\frac{k l r}{2 h t}\right)^{2} r^{\nu-2} I_{-\nu}\left(\frac{k l r}{2 h t}\right)\right\},
\end{aligned}
$$

which was obtained from (3.17) using (3.9) twice.

These properties of $g$ give us

LEMMA 3.2. The function $v$ given by (3.1) is well defined in $Q(l, 0)$, is continuously differentiable with respect to $t$ and twice continuously differentiable with respect to $x$ there, and is continuous in $\overline{Q(l, 0)} \backslash\{(x, 0) ;|x|=l\}$.

Proof. The interior regularity of $v$ follows from straightforward computations using (3.6) applied to (3.1), (3.17) and (3.18). To establish the continuity in $\overline{Q(l, 0)} \backslash\{(x, 0) ;|x|=\varphi\}$ we use (3.6) and the relation $\nu \leqslant \frac{1}{2}$ to obtain

$$
r^{-\nu} I_{-\nu}\left(\frac{h l r}{2 h t}\right) \leqslant \text { const } t^{\nu} \exp \left[\frac{k l r}{2 h t}\right]
$$

and thus we have, for $0 \leqslant r<l, t>0$

$$
\int_{0}^{t} \frac{|g(r, \tau)|}{\sqrt{t-\tau}} d t \leqslant \text { const } \int_{0}^{t} \frac{\exp \left[-k(l-r)^{2} / 4 h \tau\right]}{\tau^{1-\nu} \sqrt{t-\tau}} d \tau .
$$


This then yields

$$
\lim _{\substack{(x, t) \rightarrow\left(x^{\prime}, 0\right) \\(x, t) \in Q(l, 0)}} v(x, t)=0 \text { for }\left|x^{\prime}\right|<l .
$$

It remains to show since $g$ is continuous that (3.1) converges as $r \rightarrow l$ for $t>0$. The estimate in (3.19) is not fine enough for this purpose. Returning to (3.6) we have for $r, t>0$

$$
\begin{aligned}
I_{-\nu}\left(\frac{k l r}{2 h t}\right) & <\text { const }\left(\frac{r}{t}\right)^{-\nu} \exp \left[\frac{k l r}{2 h t}\right]\left(1+\frac{k l r}{2 h t}\right)^{\nu-1 / 2} \\
& <\text { const } \sqrt{t} r^{-\nu} \exp \left[\frac{k l r}{2 h t}\right]\left(t+\frac{k l r}{2 h t}\right)^{\nu-1 / 2} \\
& <\text { const } \sqrt{\frac{t}{r}} \exp \left[\frac{k l r}{2 h t}\right],
\end{aligned}
$$

since $\nu<\frac{1}{2}$, and hence we obtain

$$
\int_{0}^{t} \frac{g(l, \tau)}{\sqrt{t-\tau}} d \tau<\text { const } \int_{0}^{t} \frac{d \tau}{\sqrt{\tau} \sqrt{t-\tau}}=\text { const } \int_{0}^{1} \frac{d \xi}{\sqrt{\xi} \sqrt{\xi-1}} .
$$

This last integral exists and equals $\pi$.

Note that $v$ is not continuous in $\overline{Q\left(l, t_{0}\right)}$. In fact, $v(x, 0)=0$ for $|x|<l$ while for $|x|=l$ and $t>0$ sufficiently small the asymptotic estimate $I_{p}(r) \sim$ $(2 \pi r)^{-1 / 2} e^{r}$ as $r \rightarrow \infty$ shows that

$$
\begin{aligned}
\sqrt{\frac{2}{\pi}} v(x, t) & =\sqrt{\frac{k}{\pi h}} l \int_{0}^{t} \frac{\exp \left[-k l^{2} / 2 h \tau\right]}{\tau \sqrt{t-\tau}} I_{-\nu}\left(\frac{k l^{2}}{2 h \tau}\right) d \tau \\
& \sim \frac{1}{\pi} \int_{0}^{t} \frac{d \tau}{\sqrt{\tau} \sqrt{t-\tau}}=1,
\end{aligned}
$$

which implies that $v(x, t) \rightarrow \sqrt{\pi / 2}$ as $t \rightarrow 0$ when $|x|=l$. We have dropped the constant $\sqrt{2 / \pi}$ from (3.1) to simplify later calculations.

LEMMA 3.3. The function $v$ satisfies $(3.4)$ in the cylinder $Q(l, 0)$.

Proof. For $r>0$ we may differentiate under the integral to obtain

$$
\begin{aligned}
h v_{r r}+ & \frac{\beta+1-h}{r} v_{r}-k v_{t} \\
& =\text { const } \int_{0}^{t} \frac{h g_{r r}+(\beta+1-h) / r g_{r}-k g_{\tau}}{\sqrt{t-\tau}} d \tau=0 .
\end{aligned}
$$

The continuity of the functions $v_{r r}, v_{r} / r$ and $v_{t}$ gives the result also for $r=0$.

LEMMA 3.4. In $D(l, 0)$, for any $l>1$, we have

$$
v_{r r}-v_{r} / r \geqslant 0 \text {. }
$$


Proof. Note, first of all, that (3.20) is automatically satisfied with equality if $r=0$ and $t>0$. To establish (3.20) for $r>0$ we note that $v_{r r}-v_{r} / r=$ $r\left(v_{r} / r\right)$, and, from (3.17) and (3.9), that

$$
\begin{aligned}
\left(\frac{v_{r}}{r}\right)_{r}= & \text { const } \int_{0}^{t} \frac{\exp \left[-k\left(l^{2}+r^{2}\right) / 4 h t\right]}{\tau^{2} \sqrt{t-\tau}}\left(\frac{k}{2 h}\right)^{2} r^{\nu-1} \\
& \times\left\{r^{2} I_{-\nu}\left(\frac{k l r}{2 h \tau}\right)-2 l r I_{1-\nu}\left(\frac{k l r}{2 h \tau}\right)+l^{2} I_{2-\nu}\left(\frac{k l r}{2 h t}\right)\right\} d \tau .
\end{aligned}
$$

It then suffices to show that

$$
r^{2} I_{-\nu}\left(\frac{k l r}{2 h \tau}\right)-2 l r I_{1-\nu}\left(\frac{k l r}{2 h \tau}\right)+l^{2} I_{2-\nu}\left(\frac{k l r}{2 h \tau}\right)>0
$$

for $r, \tau$ in the range

$$
0<r<l, \quad 0<\tau<k(l-r)^{2} / 8 h(2-\nu) .
$$

To simplify notation we set

$$
\xi=k l r / 2 h \tau \text { and } \rho=r / l
$$

so that (3.21) and (3.22) are equivalent to

$$
\rho^{2} I_{-\nu}(\xi)-2 \rho I_{1-\nu}(\xi)+I_{2-\nu}(\xi)>0
$$

for $\rho$ and $\xi$ in the range

$$
0<\rho<1, \quad(1-\rho)^{2}>4(2-\nu) \rho \xi^{-1} .
$$

We apply (3.12) and (3.22)' to (3.23)' to obtain

$$
\begin{aligned}
\rho^{2} I_{-\nu}(\xi)- & 2 \rho I_{1-\nu}(\xi)+I_{2-\nu}(\xi) \\
= & (1-\rho)^{2} I_{2-\nu}(\xi)-2 \rho\left(I_{1-\nu}(\xi)-I_{2-\nu}(\xi)\right) \\
& +2 \rho^{2}(1-\nu) \xi^{-1} I_{1-\nu}(\xi) \\
> & \frac{4(2-\nu)}{\xi} \rho I_{2-\nu}(\xi)-2 \rho\left(I_{1-\nu}(\xi)-I_{2-\nu}(\xi)\right) \\
& +2 \rho^{2}(1-\nu) \xi^{-1} I_{1-\nu}(\xi) .
\end{aligned}
$$

Disregarding the last nonnegative term, using (3.12) again, and finally applying (3.8) we have (3.21)'

$$
\begin{aligned}
\rho^{2} I_{-\nu}(\xi)- & 2 \rho I_{1-\nu}(\xi)+I_{2-\nu}(\xi) \\
\geqslant & 2 \rho\left(I_{1-\nu}(\xi)-I_{3-\nu}(\xi)\right)-2 \rho\left(I_{1-\nu}(\xi)-I_{2-\nu}(\xi)\right) \\
& =2 \rho\left(I_{2-\nu}(\xi)-I_{3-\nu}(\xi)\right) \geqslant 0 .
\end{aligned}
$$

LEMMA 3.5. In $D(l, 0)$ we have

$$
v_{t}(x, t) \geqslant t^{-1} v(x, t) \geqslant 0 .
$$


Proof. By (3.16) and Lemma 3.2 we see that for $|x|>0$

$$
\begin{aligned}
v_{t}(x, t)= & \sqrt{\frac{k}{2 h}} l^{1-\nu} \int_{0}^{t} \frac{\exp \left[-k\left(l^{2}+r^{2}\right) / 4 h t\right]}{\tau^{2} \sqrt{t-\tau}} r^{\nu} \\
& \times\left\{\left[\frac{k\left(l^{2}+r^{2}\right)}{4 h \tau}-\frac{(\gamma+1)}{2}\right] I_{-\nu}\left(\frac{k l r}{2 h \tau}\right)\right. \\
& \left.\quad-\frac{k l r}{2 h \tau} I_{1-\nu}\left(\frac{k l r}{2 h \tau}\right)\right\} d \tau .
\end{aligned}
$$

We restrict attention to the term within braces which equals

$$
\begin{aligned}
{\left[\frac{k(l-r)^{2}}{4 h \tau}-\frac{(\gamma+1)}{2}\right] } & I_{-\nu}\left(\frac{k l r}{2 h \tau}\right) \\
& +\frac{k l r}{2 h \tau}\left[I_{-\nu}\left(\frac{k l r}{2 h \tau}\right)-I_{1-\nu}\left(\frac{k l r}{2 h \tau}\right)\right] .
\end{aligned}
$$

Since $-\nu \geqslant \frac{1}{2},(3.8)$ shows that the last term is nonnegative. For $(x, t) \in$ $D(l, 0)$ and $0<\tau \leqslant t$ we use (3.13) to note that

$$
\begin{aligned}
\frac{k(l-r)^{2}}{4 h \tau} & \geqslant \frac{k(l-r)^{2}}{4 h t} \geqslant \frac{k(l-r)^{2}}{4 h} \cdot \frac{8 h(2-\nu)}{k(l-r)^{2}}=2(2-\nu) \\
& =2+(1+\gamma)>1+\frac{(1+\gamma)}{2}
\end{aligned}
$$

so that (3.24) dominates $I_{-\nu}(k l r / 2 h \tau)$. We thus have

$$
\begin{aligned}
v_{t}(x, t) & \geqslant \sqrt{\frac{k}{2 h}} l^{1-\nu} \int_{0}^{t} \frac{\exp \left[-k\left(l^{2}+r^{2}\right) / 4 h \tau\right]}{\tau^{2} \sqrt{t-\tau}} r^{\nu} I_{-\nu}\left(\frac{k l r}{2 h \tau}\right) d \tau \\
& \geqslant \frac{1}{t} v(x, t) \geqslant 0 \text { for }|x|>0 .
\end{aligned}
$$

Continuity gives the result then for $|x|=0$.

LEMMA 3.6. In $D(l, 0)$ we have

$$
v_{r}(x, t) \geqslant 0 .
$$

Proof. Set

$$
T=k\left(l^{2}-1\right) / 8 h(2-\nu)
$$

and, for $0<t<T$, denote by $l(t)$ the radius of the cross section of $D\left(l, t_{0}\right)$ at height $t$ so that we can write $D(l, 0)=\{(x, t): 0<t<T,|x|<l(t)\}$. On the axis $x=0,0<t<T$ (3.25) holds with equality by Lemma 3.2. To obtain the result for $|x|>0$, fix $t, 0<t<T$, and consider the function 


$$
f(r)=v_{r} / r,
$$

noting that $f(0)$ is finite. Since $v$ is a solution of $(3.4)$ and $v_{t} \geqslant 0$ in $D(l, 0)$, we have

$$
\begin{aligned}
0 & \leqslant h v_{r r}+(\beta+1-h) v_{r} / r=h r\left(v_{r} / r\right)_{r}+(\beta+1)\left(v_{r} / r\right) \\
& =h r f^{\prime}(r)+(\beta+1) f(r) \text { for } 0<r<l(t)
\end{aligned}
$$

which implies that

$$
\left(r^{(\beta+1) / h} f(r)\right)^{\prime} \geqslant 0 \text { for } 0<r<l(t) .
$$

Noting that $(\beta+1) h^{-1}>0$ and integrating from zero to $r$ we have

$$
r^{(\beta+1) / h} f(r) \geqslant 0 \text { for } 0<r<l(t)
$$

which implies (3.25).

LEMMA 3.7. There is a positive constant $B_{\nu}$ depending only upon $\nu$ such that

$$
v(x, t) \geqslant B_{\nu} \text { for all }(x, t) \in P(l, 0) .
$$

Proof. For $r=|x|>0$ we can make the change of variable $\xi=k l r / 2 h \tau$ in (3.1) to obtain

$$
\begin{aligned}
v(x, t) & =\sqrt{\frac{k}{2 h}} l^{1-\nu} \int_{k l r / 2 h t}^{\infty} \frac{\exp \left[-\left(l^{2}+r^{2}\right) \xi / 2 l r\right]}{(k l r / 2 h \xi) \sqrt{t-k l r / 2 h \xi}} r^{\nu} I_{-\nu}(\xi) \frac{k l r}{2 h \xi^{2}} d \xi \\
& =\sqrt{\frac{k l r}{2 h t}}\left(\frac{r}{l}\right)^{\nu-1 / 2} \int_{k l r / 2 h t}^{\infty} \frac{\exp \left[-\left(l^{2}+r^{2}\right) \xi / 2 l r\right]}{\sqrt{\xi} \sqrt{\xi-k l r / 2 h t}} I_{-\nu}(\xi) d \xi .
\end{aligned}
$$

To simplify notation we set

$$
\delta=k l r / 2 h t \text { and } \rho=r / l
$$

so that we have

$$
\begin{aligned}
& v(x, t)=\sqrt{\delta} \rho^{\nu-1 / 2} \int_{\delta}^{\infty} \frac{\exp \left[-\left(1+\rho^{2}\right) \xi / 2 \rho\right]}{\sqrt{\xi} \sqrt{\xi-\delta}} I_{-\nu}(\xi) d \xi \\
&=\sqrt{\delta} \rho^{\nu-1 / 2} \int_{1}^{\infty} \frac{\exp \left[-\left(1+\rho^{2}\right) \delta \eta / 2 \rho\right]}{\sqrt{\eta} \sqrt{\eta-1}} I_{-\nu}(\delta \eta) d \eta \\
& \quad \text { for } \delta>0,0<\rho<1 .
\end{aligned}
$$

On $P(l, 0)$ we have

$$
\delta=\frac{4(2-\nu) \rho}{(1-\rho)^{2}} \quad \text { with } \frac{1}{l} \leqslant \rho<1
$$

so that for $(x, t) \in P(l, 0)$ we obtain 


$$
\begin{aligned}
& v(x, t)=\sqrt{\frac{4(2-\nu)}{(1-\rho)^{2}}} \rho^{\nu} \int_{1}^{\infty} \frac{\exp \left[-\frac{\left(1+\rho^{2}\right) \eta}{2 \rho} \cdot \frac{4(2-\nu) \rho}{(1-\rho)^{2}}\right]}{\sqrt{\eta} \sqrt{\eta-1}} \\
& \times I_{-\nu}\left(\frac{4(2-\nu) \rho \eta}{(1-\rho)^{2}}\right) d \eta \\
& =\int_{1}^{\infty} \frac{\exp [-2(2-\nu) \eta]}{\sqrt{\eta} \sqrt{\eta-1}}\left[\sqrt{\frac{4(2-\nu)}{(1-\rho)^{2}}} \rho^{\nu} \exp \left[-\frac{4(2-\nu) \rho \eta}{(1-\rho)^{2}}\right]\right. \\
& \left.\times I_{-\nu}\left(\frac{4(2-\nu) \rho \eta}{(1-\rho)^{2}}\right)\right] d \eta \text {. }
\end{aligned}
$$

We use (3.6) to see that

$$
\begin{gathered}
\rho^{\nu} \sqrt{\frac{4(2-\nu)}{(1-\rho)^{2}}} \exp \left[-\frac{4(2-\nu) \rho \eta}{(1-\rho)^{2}}\right] I_{-\nu}\left(\frac{4(2-\nu) \rho \eta}{(1-\rho)^{2}}\right) \\
>\lambda_{-\nu} \frac{\left(4(2-\nu) \rho \eta /(1-\rho)^{2}\right)^{-\nu} \rho^{\nu} \sqrt{4(2-\nu) /(1-\rho)^{2}}}{\left(1+4(2-\nu) \rho \eta /(1-\rho)^{2}\right)^{1 / 2-\nu}} \\
=\frac{\lambda_{-\nu}[4(2-\nu)]^{1 / 2-\nu} \eta^{-\nu}}{\left((1-\rho)^{2}+4(2-\nu) \rho \eta\right)^{1 / 2-\nu}}
\end{gathered}
$$

The companion estimate, which we shall need in Lemma 3.8,

$$
\begin{gathered}
\rho^{\nu} \sqrt{\frac{4(2-\nu)}{(1-\rho)^{2}}} \exp \left[\frac{4(2-\nu) \rho \eta}{(1-\rho)^{2}}\right] I_{-\nu}\left(\frac{4(2-\nu) \rho \eta}{(1-\rho)^{2}}\right) \\
<\Lambda_{-\nu} \frac{[4(2-\nu)]^{1 / 2-\nu} \eta^{-\nu}}{\left((1-\rho)^{2}+4(2-\nu) \rho \eta\right)^{1 / 2-\nu}}
\end{gathered}
$$

follows in the same manner. Since $(1-\rho)^{2}+4(2-\nu) \rho \eta$ is increasing for $0<\rho<1$ and $\nu<\frac{1}{2}$ we obtain the bounds

$$
\begin{aligned}
\frac{\lambda_{-\nu}}{\sqrt{\eta}} & \leqslant \rho^{\nu} \sqrt{\frac{4(2-\nu)}{(1-\rho)^{2}}} \exp \left[-\frac{4(2-\nu) \rho \eta}{(1-\rho)^{2}}\right] I_{-\nu}\left(\frac{4(2-\nu) \rho \eta}{(1-\rho)^{2}}\right) \\
& \leqslant \frac{\Lambda_{-\nu}[4(2-\nu)]^{1 / 2-\nu}}{\eta^{\nu}}
\end{aligned}
$$


valid for $0<\rho<1$ and $\eta \geqslant 1$. We conclude from the lower estimate that

$$
v(x, t) \geqslant \lambda_{-\nu} \int_{1}^{\infty} \frac{\exp [-2(2-\nu) \eta]}{\eta \sqrt{\eta-1}} d \eta \equiv B_{\nu} \quad \text { on } P(l, 0) .
$$

LEMMA 3.8. There is a positive constant $A_{\nu}$ depending only on $\nu$ such that

$$
v(x, t)<A_{\nu} \exp \left(-\frac{k(l-|x|)^{2}}{8 h t}\right) \text { in } D(l, 0) \text {. }
$$

Proof. It suffices by continuity to establish (3.30) for $|x|>0$. We use the representation (3.28)

$$
v(x, t)=\sqrt{\delta} \rho^{\nu-1 / 2} \int_{\delta}^{\infty} \frac{\exp \left[-\left(1+\rho^{2}\right) \xi / 2 \rho\right]}{\sqrt{\xi} \sqrt{\xi-\delta}} I_{-\nu}(\xi) d \xi
$$

in terms of the variables $\delta$ and $\rho$ of (3.27). For $\xi>\delta$ we have

$$
\begin{aligned}
2\left(1+\rho^{2}\right) \xi & =(1-\rho)^{2} \xi+(1+\rho)^{2} \xi \\
& \geqslant(1-\rho)^{2} \delta+(1+\rho)^{2} \xi
\end{aligned}
$$

so that we obtain the preliminary estimate

$$
\begin{aligned}
v(x, t) \leqslant & \exp \left[-\frac{(1-\rho)^{2} \delta}{4 \rho}\right] \\
& \times\left\{\int_{1}^{\infty} \frac{\exp \left[-(1+\rho)^{2} \delta \eta / 4 \rho\right]}{\sqrt{\eta} \sqrt{\eta-1}} \rho^{\nu-1 / 2} \sqrt{\delta} I_{-\nu}(\delta \eta) d \eta\right\} \\
\equiv & \exp \left[-\frac{(1-\rho)^{2} \delta}{4 \rho}\right] V(\delta, \rho) .
\end{aligned}
$$

Since $k(l-r)^{2}(8 h t)^{-1}=(1-\rho)^{2} \delta(4 \rho)^{-1}$ the desired result will follow if we can show that

$$
V(\delta, \rho)<A_{\nu} \text { for } 0<\rho<1 \text { and } \delta>4(2-\nu) \rho /(1-\rho)^{2} .
$$

We shall first estimate $V$ with respect to $\delta$ for a fixed $0<\rho<1$ and then use the upper bound in (3.29) to obtain (3.31). Consider the function

$$
\omega(\delta)=\sqrt{\delta} \exp \left(-\frac{(1+\rho)^{2} \delta \eta}{4 \rho}\right) I_{-\nu}(\delta \eta)
$$

for $\delta>4(2-\nu) \rho(1-\rho)^{-2}$ with $\eta \geqslant 1$ and $0<\rho<1$ fixed. Differentiation with respect to $\delta$ and the use of (3.11) yields 


$$
\frac{d \omega}{d \delta}=\sqrt{\delta} \exp \left(-\frac{(1+\rho)^{2} \delta \eta}{4 \rho}\right) g(\delta)
$$

where $G(\delta)$ denotes the expression

$$
\left[\frac{\left(\frac{1}{2}-\nu\right)}{\delta}-\frac{(1+\rho)^{2} \eta}{4 \rho}\right] I_{-\nu}(\delta \eta)+\eta I_{1-\nu}(\delta \eta) .
$$

For $\eta \geqslant 1, \nu \leqslant \frac{1}{2}$ and using the bound on $\delta$ we have

$$
\begin{aligned}
\frac{\left(\frac{1}{2}-\nu\right)}{\delta}-\frac{(1+\rho)^{2} \eta}{4 \rho} & \leqslant\left[\frac{\left(\frac{1}{2}-\nu\right)}{\delta}-\frac{(1+\rho)^{2}}{4 \rho}\right] \eta \\
& \leqslant\left[\frac{\left(\frac{1}{2}-\nu\right)(1-\rho)^{2}}{(2-\nu) 4 \rho}-\frac{(1+\rho)^{2}}{4 \rho}\right] \eta \leqslant-\eta .
\end{aligned}
$$

With this estimate and (3.8) we conclude that

$$
g(\delta) \leqslant \eta\left[I_{1-\nu}(\delta \eta)-I_{-\nu}(\delta \eta)\right] \leqslant 0,
$$

and thus $\omega$ is a nonincreasing function of $\delta$. It follows that

$$
\begin{aligned}
V(\delta, \rho) & =\rho^{\nu-1 / 2} \int_{1}^{\infty} \frac{\omega(\delta)}{\sqrt{\eta} \sqrt{\eta-1}} d \eta \\
& \leqslant \rho^{\nu-1 / 2} \int_{1}^{\infty} \frac{\omega\left(4(2-\nu) \rho /(1-\rho)^{2}\right)}{\sqrt{\eta} \sqrt{\eta-1}} d \eta \\
& =\int_{1}^{\infty} \frac{\exp [-(2-\nu) \eta]}{\sqrt{\eta} \sqrt{\eta-1}}\left[\rho^{\nu} \sqrt{\frac{4(2-\nu)}{(1-\rho)^{2}}} \exp \left[-\frac{4(2-\nu) \rho \eta}{(1-\rho)^{2}}\right]\right. \\
& \left.\times I_{-\nu}\left(\frac{4(2-\nu) \rho \eta}{(1-\rho)^{2}}\right)\right] d \eta .
\end{aligned}
$$

We finally use (3.29) to obtain

$$
V(\delta, \rho) \leqslant \Lambda_{-\nu}[4(2-\nu)]^{1 / 2-\nu} \int_{1}^{\infty} \frac{\exp [-(2-\nu) \eta]}{\eta^{1 / 2+\nu} \sqrt{\eta-1}} d \eta \equiv A_{\nu} .
$$

IV. Proof of Theorem I. To establish Theorem I we use the basic technique of Täcklind suitably modified to take into account the more complicated sets with which we are working. We divide the proof into two cases depending upon whether or not the function $p$ tends to infinity with $r$.

Case $1 . \lim \inf _{r \rightarrow \infty} p(r)<\infty$. In this case there is a constant $P, 0<P<$ 
$\infty$, and a sequence $\left\{r_{j}\right\}$ with $r_{j} \rightarrow \infty$ such that $p\left(r_{j}\right)<P$. Let $\varepsilon, T>0$ and $l>1$ be given and choose $l^{*}=r_{j}$ with $j$ so large that $\{(x, t) \in \Omega:|x| \leqslant l$ and $t<T\} \subseteq \Omega\left(l^{*}, 0\right)$ and

$$
M C \exp \left(\mu l^{*} P-\frac{k\left(l^{*}-l\right)^{2}}{8 h T}\right)<\varepsilon .
$$

Here $M, \mu$ and $C$ are the constants from the growth condition and property (T).

From the growth condition we have

$$
\begin{aligned}
\limsup _{\substack{(x, t) \rightarrow S\left(l^{*}, 0\right) \\
(x, t) \in \Omega\left(l^{*}, 0\right)}} u(x, t) & \leqslant \limsup _{\substack{(x, t) \rightarrow S\left(l^{*}, 0\right) \\
(x, t) \in \Omega\left(l^{*}, 0\right)}} M \exp (\mu|x| p(|x|)) \\
& \leqslant M \exp \left(\mu l^{*} p\left(l^{*}\right)\right) \leqslant M \exp \left(\mu l^{*} P\right)
\end{aligned}
$$

since $r p(r)$ is nondecreasing. Applying property $(\mathrm{T})$ with $k_{1}=0$ and $K_{2}=$ $M \exp \left(\mu L^{*} P\right)$ in $\Omega\left(l^{*}, 0\right)$ we have

$$
u(x, t) \leqslant M C \exp \left(\mu l^{*} P-\frac{k\left(l^{*}-|x|\right)^{2}}{8 h t}\right)<\varepsilon
$$

for $(x, t) \in \Omega$ with $|x|<l$ and $t<T$.

Case 2. $\lim _{r \rightarrow \infty} p(r)=+\infty$. In this case we obtain the result in the set $\{(x, t) \in \Omega:|x|<l, t<T\}$ by applying the growth condition and property (T) iteratively on an appropriately chosen finite sequence of sets $\Omega\left(l_{i}, t_{i}\right)$ to estimate $u(x, t)$ for $(x, t) \in \Omega$ and $|x|<l$ for progressively larger values of $t$. Täcklind's approach was similar but simpler since he had only to deal with cylinders.

The choice of the $l_{i}$ and the $t_{i}$ is related to the properties of $p$ and the divergence of the integral $\int^{\infty} p^{-1}(r) d r$. For this purpose set

$$
\bar{p}(r)=\inf _{s>r} p(s) \text {. }
$$

Choose $l_{0}>1$ such that $\bar{p}\left(l_{0}\right)=p\left(l_{0}\right)$ and $(1+\mu) l_{0} p\left(l_{0}\right)>2-\nu$. For $i=$ $1,2, \ldots$, let $l_{i}$ be the smallest number satisfying

(i) $l_{i} \geqslant 2 l_{i-1}$,

(ii) $\bar{p}\left(l_{i}\right)=p\left(l_{i}\right)$.

We shall also need the auxiliary sequence $\left\{b_{i}\right\}$ defined by

$$
b_{i}=\frac{k\left(l_{i}-l_{i-1}\right)^{2}}{8 h(\mu+1) l_{i} p\left(l_{i}\right)}, \quad i=1,2, \ldots
$$

The divergence of the series $\sum b_{i}$ will be necessary later for the choice of $l^{*}$ and the sequence $\left\{t_{i}\right\}$. The estimates

$$
\frac{k l_{i}}{32 h(1+\mu) p\left(l_{i}\right)} \leqslant b_{i} \leqslant \frac{k l_{i}}{8 h(1+\mu) p\left(l_{i}\right)},
$$


which follow since $l_{i} \geqslant 2 l_{i-1}$, show us that the two series $\Sigma b_{i}$ and $\Sigma l_{i} / p\left(l_{i}\right)$ converge or diverge together. We will establish the divergence of the series $\sum b_{i}$ and also the equivalence of our condition, $\int^{\infty} p^{-1}(r) d r=+\infty$, and Täcklind's, $\int^{\infty} \bar{p}^{-1}(r) d r=+\infty$, by showing that, when $r p(r)$ is nondecreasing, these two integrals and the series $\Sigma l_{i} / p\left(l_{i}\right)$ all converge or diverge simultaneously.

We note, from the definition of $\bar{p}$, that $p(r) \geqslant \bar{p}(r)$ for all $r>1$ and if $l_{i}>2 l_{i-1}$ then $\bar{p}(r) \equiv p\left(l_{i}\right)$ for $2 l_{i-1} \leqslant r \leqslant l_{i}$. With this we see that

$$
\begin{aligned}
\int_{l_{i-1}}^{l_{i}} \frac{d r}{\bar{p}(r)} & =\int_{l_{i-1}}^{2 l_{i-1}} \frac{d r}{\bar{p}(r)}+\int_{2 l_{i-1}}^{l_{i}} \frac{d r}{\bar{p}(r)} \\
& \leqslant \frac{l_{i-1}}{p\left(l_{i-1}\right)}+\frac{l_{i}-2 l_{i-1}}{p\left(l_{i}\right)} \\
& \leqslant \frac{l_{i-1}}{p\left(l_{i-1}\right)}+\frac{l_{i}}{p\left(l_{i}\right)}
\end{aligned}
$$

so that we have

$$
\begin{aligned}
\int_{l_{n}}^{l_{N}} \frac{d r}{\bar{p}(r)} & =\sum_{i=n+1}^{N} \int_{l_{i-1}}^{l_{i}} \frac{d r}{\bar{p}(r)} \\
& \leqslant \sum_{i=n+1}^{N} \frac{l_{i-1}}{p\left(l_{i-1}\right)}+\frac{l_{i}}{p\left(l_{i}\right)}<2 \sum_{i=n}^{N} \frac{l_{i}}{p\left(l_{i}\right)} .
\end{aligned}
$$

The lower bound

$$
\int_{l_{i-1}}^{l_{i}} \frac{d r}{\bar{p}(r)} \geqslant \frac{l_{i}-l_{i-1}}{p\left(l_{i}\right)} \geqslant \frac{l_{i}}{2 p\left(l_{i}\right)}
$$

is clear since $\bar{p}$ is nondecreasing. These estimates combine to give

$$
\frac{1}{2} \sum_{i=n+1}^{N} \frac{l_{i}}{p\left(l_{i}\right)}<\int_{l_{n}}^{l_{N}} \frac{d r}{\bar{p}(r)}<2 \sum_{i=n}^{N} \frac{l_{i}}{p\left(l_{i}\right)}
$$

from which it follows that the series $\Sigma l_{i} / p\left(l_{i}\right)$ and the integral $\int^{\infty} \bar{p}^{-1}(r) d r$ converge or diverge together. Since $\bar{p}(r) \leqslant p(r)$ we have

$$
\int_{l_{n}}^{l_{N}} \frac{d r}{p(r)}<\int_{l_{n}}^{l_{N}} \frac{d r}{\bar{p}(r)}<2 \sum_{i=n}^{N} \frac{l_{i}}{p\left(l_{i}\right)} .
$$

If we include our additional condition that $r p(r)$ is nondecreasing then we also have the lower estimate

$$
\int_{l_{i-1}}^{l_{i}} \frac{d r}{p(r)}=\int_{l_{i-1}}^{l_{i}} \frac{r d r}{r p(r)} \geqslant \frac{l_{i}^{2}-l_{i-1}^{2}}{l_{i} p\left(l_{i}\right)} \geqslant \frac{3 l_{i}}{8 p\left(l_{i}\right)}
$$

and hence the integral $\int^{\infty} p^{-1}(r) d r$ and the series $\Sigma l_{i} / p\left(l_{i}\right)$ converge or diverge together since 


$$
\frac{3}{8} \sum_{i=n+1}^{N} \frac{l_{i}}{p\left(l_{i}\right)} \leqslant \int_{l_{n}}^{l_{N}} \frac{d r}{p(r)} \leqslant 2 \sum_{i=n}^{N} \frac{l_{i}}{p\left(l_{i}\right)} .
$$

We can now choose $l^{*}$ and the sequence $\left\{t_{i}\right\}$. Let $\varepsilon, T>0$ and $l>1$ be given and choose $n$ so large that $\{(x, t) \in \Omega ;|x|<l, t<T\} \subseteq \Omega\left(l_{n-1}, 0\right)$ and

$$
\frac{C M}{\exp \left(l_{n} p\left(l_{n}\right)\right)-1}<\varepsilon
$$

Here $M$ and $C$ are the constants from the growth condition and proper!, $m$ Choose $N$ so that

$$
\sum_{i=n}^{N} b_{i} \geqslant T
$$

which can be done since $\sum b_{i}$ diverges and set $l^{*}=l_{N}$. We define $t_{N}=0 a^{-1}$

$$
t_{i}=\sum_{j=i+1}^{N} b_{j} \text { for } i=n-1, n, \ldots, N-1 .
$$

We wish to apply property (T) iteratively in the sets $\Omega\left(l_{N}\right.$, $\Omega\left(l_{N-1}, t_{N-1}\right) \supseteq \cdots \supseteq \Omega\left(l_{n-1}, t_{n-1}\right)$. We first must establish the set containment $\Omega\left(l_{i}, t_{i}\right) \supseteq \Omega\left(l_{i-1}, t_{i-1}\right)$ for $i=n, \ldots, N$. Since $l_{i}>l_{i-1}$ it suffices to show that

$$
t_{i-1}+\frac{k\left(l_{i-1}-r\right)^{2}}{8 h(2-\nu)}<t_{i}+\frac{k\left(l_{i}-r\right)^{2}}{8 h(2-\nu)} \text { for } 0<r<l_{i-1} \text {. }
$$

We note that

$$
2-\nu<(1+\mu) l_{0} p\left(l_{0}\right)<(1+\mu) l_{i} p\left(l_{i}\right)
$$

since $r p(r)$ is nondecreasing, and hence we have

$$
\begin{aligned}
t_{i-1}+\frac{k\left(l_{i-1}-r\right)^{2}}{8 h(2-\nu)} & =t_{i}+b_{i}+\frac{k\left(l_{i-1}-r\right)^{2}}{8 h(2-\nu)} \\
& <t_{i}+\frac{k}{8 h(2-\nu)}\left[\left(l_{i-1}-r\right)^{2}+\left(l_{i}-l_{i-1}\right)^{2}\right] .
\end{aligned}
$$

The inequality (4.1) then follows from the estimate

$$
\left(l_{i}-r\right)^{2}-\left(l_{i-1}-r\right)^{2}=\left(l_{i}-l_{i-1}\right)\left(l_{i}+l_{i-1}-2 r\right)>\left(l_{i}-l_{i-1}\right)^{2}
$$

for $0 \leqslant r \leqslant l_{i-1}$.

We now apply property (T) first in $\Omega\left(l^{*}, 0\right)=\Omega\left(l_{N}, t_{N}\right)$. As in Case 1 we take

$$
K_{1}=0 \quad \text { and } \quad K_{2}=M \exp \left(\mu l_{N} p\left(l_{N}\right)\right)
$$

to obtain the estimate 


$$
u(x, t) \leqslant C M \exp \left(\mu l_{N} p\left(l_{N}\right)-\frac{k\left(l_{N}-|x|\right)^{2}}{8 h t}\right) \text { in } \Omega\left(l_{N}, t_{N}\right) .
$$

Restricting attention now to those $(x, t) \in \Omega\left(l_{N}, t_{N}\right)$ with $|x| \leqslant l_{N-1}$ and $t \leqslant t_{N-1}=b_{N}$, we have

$$
\begin{aligned}
u(x, t) & \leqslant C M \exp \left(\mu l_{N} p\left(l_{N}\right)-\frac{k\left(l_{N}-|x|\right)^{2}}{8 h b_{N}}\right) \\
& =C M \exp \left(-l_{N} p\left(l_{N}\right)\right) .
\end{aligned}
$$

We next apply property (T) in $\Omega\left(l_{N-1}, t_{N-1}\right)$ with

$$
K_{1}=C M \exp \left(-l_{N} p\left(l_{N}\right)\right) \text { and } K_{2}=M \exp \left(\mu l_{N-1} p\left(l_{N-1}\right)\right)
$$

to obtain

$$
\begin{aligned}
u(x, t) \leqslant & C M \exp \left(-l_{N} p\left(l_{N}\right)\right) \\
& +C M \exp \left(\mu l_{N-1} p\left(l_{N-1}\right)-\frac{k\left(l_{N-1}-|x|\right)^{2}}{8 h\left(t-t_{N-1}\right)}\right)
\end{aligned}
$$

in $\Omega\left(l_{N-1}, t_{N-1}\right)$. Restricting attention to those $(x, t) \in \Omega\left(l_{N-1}, t_{N-1}\right)$ with $|x|<l_{N-2}$ and $t \leqslant t_{N-2}=t_{N-1}+b_{N-1}$ we have

$$
\begin{aligned}
u(x, t) \leqslant & C M \exp \left\{-l_{N} p\left(l_{N}\right)\right\} \\
& +C M \exp \left\{u l_{N-1} p\left(l_{N-1}\right)-\frac{k\left(l_{N-1}-l_{N-2}\right)^{2}}{8 h b_{N-1}}\right\} \\
= & C M \exp \left\{-l_{N} p\left(l_{N}\right)\right\}+C M \exp \left\{-l_{N-1} p\left(l_{N-1}\right)\right\} .
\end{aligned}
$$

In particular, from (4.2) and (4.3), we have the estimate

$$
u(x, t) \leqslant C M\left(\exp \left\{-l_{N} p\left(l_{N}\right)\right\}+\exp \left\{-l_{N-1} p\left(l_{N-1}\right)\right\}\right)
$$

in the set $\left\{(x, t) \in \Omega:|x|<l, t \leqslant t_{N-2}\right\} \cup\left\{(x, t) \in \Omega\left(l_{N-1}, t_{N-1}\right):|x| \leqslant\right.$ $\left.l_{N-2}, t \leqslant t_{N-2}\right\}$. If we continue in this fashion then, in general, we obtain the estimate, for $i=N, N-1, \ldots, n$,

$$
u(x, t) \leqslant C M \sum_{j=i}^{N} \exp \left\{-l_{j} p\left(l_{j}\right)\right\}
$$

valid in the set $\left\{(x, t) \in \Omega:|x|<l, t \leqslant t_{i-1}\right\} \cup\left\{(x, t) \in \Omega\left(l_{i}, t_{i}\right):|x| \leqslant l_{i-1}\right.$, $\left.t \leqslant t_{i-1}\right\}$. In particular, taking $i=n$ we have

$$
u(x, t) \leqslant C M \sum_{j=n}^{N} \exp \left\{-l_{j} p\left(l_{j}\right)\right\} \text { for }(x, t) \in \Omega,|x|<l, t<T,
$$

since $l_{n-1}>l$ and $t_{n-1} \geqslant T$ by construction. Applying the inequalities 


$$
\begin{gathered}
p\left(l_{i}\right)=\bar{p}\left(l_{i}\right) \geqslant \bar{p}\left(l_{n}\right)=p\left(l_{n}\right) \text { for } i=n, n+1, \ldots, N, \\
l_{n+j} \geqslant 2 l_{n+j-1} \geqslant \cdots \geqslant 2^{j} l_{n} \geqslant(1+j) l_{n} \text { for } j=0,1,2, \ldots,
\end{gathered}
$$

we obtain the result

$$
\begin{aligned}
u(x, t) & \leqslant C M \sum_{j=n}^{N} \exp \left\{-l_{j} p\left(l_{j}\right)\right\} \leqslant C M \sum_{j=1}^{N-n+1} \exp \left\{-j l_{n} p\left(l_{n}\right)\right\} \\
& \leqslant C M \sum_{j=1}^{\infty}\left(\exp \left\{-l_{n} p\left(l_{n}\right)\right\}\right)^{j}=\frac{C M}{\exp \left(l_{n} p\left(l_{n}\right)\right)-1}<\varepsilon .
\end{aligned}
$$

\section{BIBLIOGRAPHY}

1. T. Carleman, Les fonctions quasianalytiques, Coll. Borel, Paris, 1926.

2. E. Holmgren, Sur les solutions quasianalytiques de l'équation de la chaleur, Ark. Mat. 18, (9) (1924), 1-9.

3. M. Krzyański, Sur les solutions des équations du type parabolique determinées dans une région illimité, Bull. Amer. Math. Soc. 47 (1941), 911-915.

4. C. Pucci, Operatori ellittici estremanti, Ann. Mat. Pura Appl. 72 (1966), 141-170.

5. P. C. Rosenbloom and D. V. Widder, A temperature function that vanishes initially, Amer. Math. Monthly 65 (1958), 607-609.

6. S. Täcklind, Sur les classes quasianalytiques des solutions des équations aux derivées partielles du type parabolique, Nova Acta Soc. Sci. Upsal. (4) 10 (1936), 1-57.

7. A. N. Tychonoff, Uniqueness theorems for the heat equation, Mat. Sb. 42 (1935), 199-216.

8. G. N. Watson, Treatise on the theory of Bessel functions, Cambridge Univ. Press, London, 1944.

9. G. N. Zolotarev, The uniqueness of the solution of the Cauchy problem for systems parabolic in the sense of I. G. Petrowski, Izv. Vysš Ucebn. Zaved. Matematika 2 (1958), 118-135. (Russian)

Department of Mathematics, University of California, Riverside, California 92502

Current address: Milliman and Robertson, Inc., Consulting Actuaries, 251 S. Lake Ave., Suite 400, Pasadena, California 91101 